\title{
A PSO Enable Multi-Hop Clustering Algorithm for VANET
}

\author{
Ankit Temurnikar, Bhagwant University, India \\ (iD) https://orcid.org/0000-0002-0416-5289 \\ Pushpneel Verma, Bhagwant University, India \\ Gaurav Dhiman, Government Bikaram College of Commerce, India
}

\begin{abstract}
VANET (vehicle ad-hoc network) is an emerging technology in today's intelligent transport system. In VANET, there are many moving nodes, which are the vehicle running on the road. They communicate with each other to provide the information to drivers regarding the road condition, traffic, weather, and parking. VANET is a kind of network where moving nodes talk with each other with the help of equipment. There are various other things that also make VANET complete like OBU (onboard unit), RSU (road aside unit), and CA (certificate authority). In this paper, a new PSO enable multihop technique is proposed that helps VANET to select the best route and find the stable cluster head and remove the malicious node from the network to avoid false messaging. The false can occur when there is a malicious node in a network. Clustering is a technique for making a group of the same type of node. This paper is based on PSO-enabled clustering and its importance in VANET. While using this approach in VANET, it has increased the $20 \%$ packet delivery ratio.
\end{abstract}

\section{KEYWORDS}

Clustering, Multi-Hop, Particle Swarm Optimization (PSO), Security

\section{INTRODUCTION}

Vehicle Ad-hoc Network one of the emerging technologies in the field of ITS (Intelligent Transport System). There are two classifications of this network: MANET and VANET. VANET plays an essential role in the area of ITS. A further category of VANET is V2V (Vehicle to Vehicle), V2I (Vehicle to infrastructure) and Hybrid V2I and V2V both. This research takes advantage of this communication. The main aim of VANET is to avoid the collision, share the traffic information and efficiently manage the available resource. VANET vehicles communicate with each other to share important information available. The vehicle is sharing information for communication with each other for solving the purpose of an intelligent transport system. Figure 1 shows the VANET architecture. It shows obvious how VANET communication takes place with the help of RSU, the Internet and OBU, how these devices communicate with each other. 


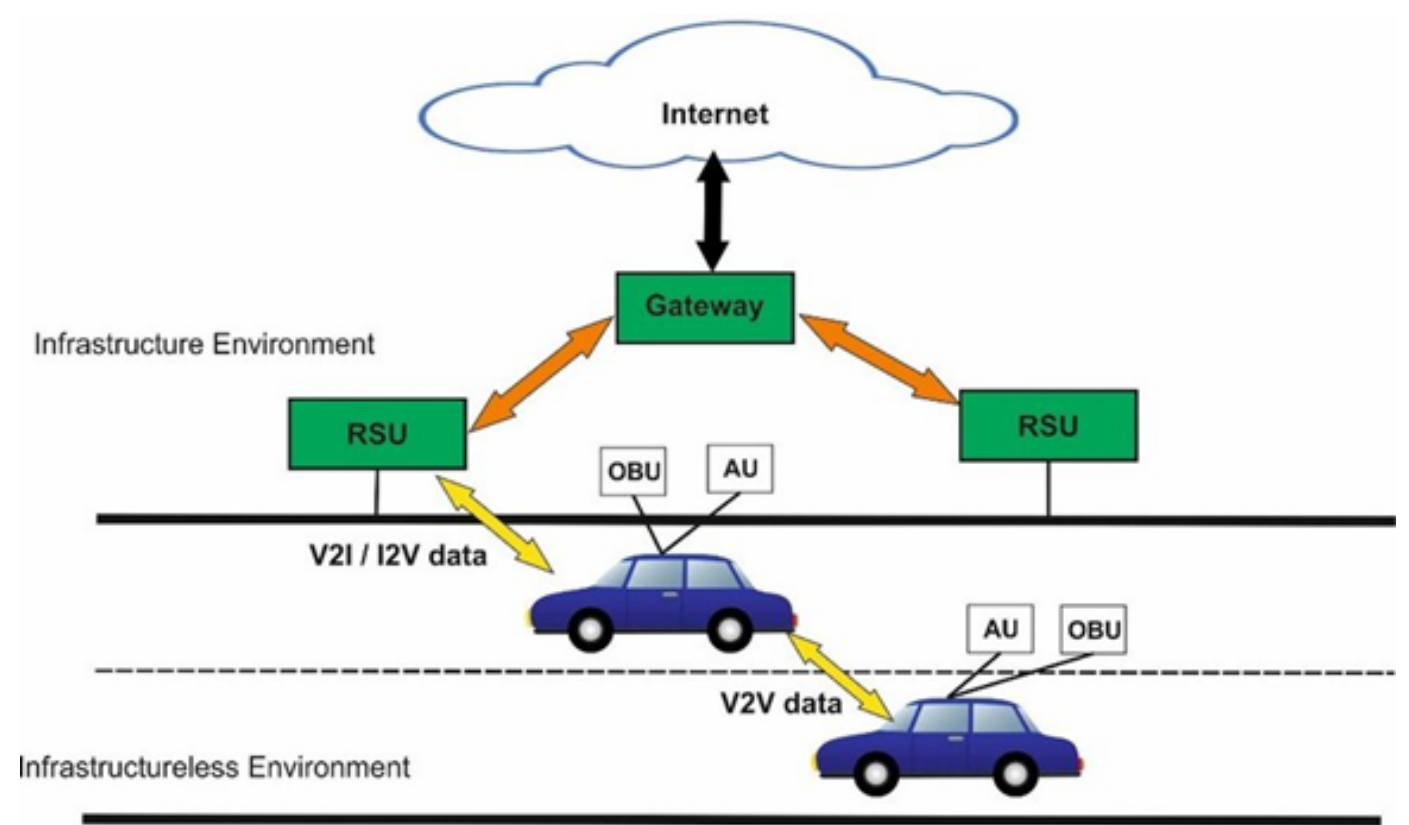

\section{CLUSTERING}

Clustering is a method of grouping the vehicle based on some predefined metrics such as velocity, density, and direction. Clustering is one of the control mechanisms in VANET. VANET is a MANET subclass, and many of the clustering technique is derived from MANET for VANET. Clustering in VANET has a highly dynamic topology that why most of the clustering algorithm is consider velocity and direction as an essential parameter for clustering. Clustering is a technique where each Cluster Head $(\mathrm{CH})$ has a Cluster member (CM) and CG (cluster gateway). In clustering, each cluster member can become a cluster head $(\mathrm{CH})$, but one can become a $\mathrm{CH}$ due to some condition in the algorithm.

\subsection{PSO (Particle Swarm Optimization)}

PSO is a heuristic algorithm for optimization. IT was expected to work with a nonlinear persistent optimization issue. It is a technique based upon swarm movement and intelligence enrage from the social behavior of birds gathering. Flocking can acquire the optimal result in this algorithm ever particle searches to scan the optimal solution with its speed. Every moving particle has an N-dimensional space that appropriately modifies its flying (Kumar et al., 2018).

The PSO algorithm can understand deeply by described following step below

- Randomly, instate the swarm with the level of the Earth heading confidential and independent location vectors.

- $\quad$ Providing a sensible vector of velocity to every practical available in network.

- Tap and make record of the fitness of each people.

- Verify the optimal implementation of molecules in the social market.

- Update velocity and position vectors as shown in (6) and (7) for each particle.

- Discrete the vector of the location.

- If any available iota flies beyond the potential arrangement space, return the molecule to its best possible and available solution before the time has come. 
- Repeat step 1 - 7 until the minute the most unmistakable number of cycles has hit (Limbasiya et al., 2018).

\section{LITERATURE REVIEW}

After an initial overview of related work, we examine specific prior work related to the PSO algorithm used in VANET. There are many types of research works based on PSO. Some of them are reviewed in the literature.

Husnain et al. (2017) research work focuses on clustering in VANET. There is a Comparatives study of PSO based algorithm. Several algorithms have been discovered and applied in VANET clustering. The study of CL PSO and MOPSO has been done, and there is a comparative study based on transmission range and number of clusters in the VANET environment. In class, the optimal number of sets is found in the maximum transmission range.

Dhurandher et al. (2019) Proposed a geometric shape based PSO approach for Routing. GPSV proposed technique uses the angle between the node and determines the best suitable route to transfer the data. For the possible shape, simulation has been done in the GPSV protocol. Circular, sine, and lane wave is used to test the swarm formation. Simulation and results are shown in throughput, end to end delay, and packet delivery ration.

Rawat et al. (2017) Presented a security scheme based on IOV (vehicle of the internet). IOV is one new current scenario technology. Since IOV provides multiple applications such as road safety, traffic updates and infotainment through information transmission. The proposed work describes data identification strategies for falsifying attacks with hashers to improve network protection and efficiency. The suggested scheme also points out the clustering method for reducing traffic congestion time. The proposed algorithm solution eliminates IOV attacks and produces a higher performance with less delay.

Waheb et al. (2016) has suggested a centralized cluster head method for intrusion detection. The cluster head tracks data for the cluster member node in the proposed job. The cluster administrators are responsible for creating the IDS and gathered data to delete the malicious node from the VANET network. If the malicious node is identified, this information is transmitted to other heads of clusters. This strategy focused on cluster head dissolves due to the high mobility of VANET nodes. The suggested scheme restricts each node's obligations due to the cluster head base. The downside of the proposed method is that this strategy has a low detection rate and high detection time in the dense network, and additional $\mathrm{CH}$ debts often degrade the network performance.

Kosuke Ozera et al. (2018) proposes the Fuzzy-based cluster management (FBCMS) cluster approach in VANET. Two models called FBCMS1 and FBCMS2 for clustering are provided in the proposed architecture in VANET. This method mainly involves three points rpm, protection and relative acceleration of the group input parameters to choose the cluster head. The FBCMS1 considers and uses a new parameter called the connectivity standard in the solution. The FBCMS2 versions include the fuzzy logic controller's trapezoidal and three-way membership features. The consequence of the suggested solution with a high GS, SC, RA value is the vehicle's broad connectivity, and a stable cluster head is chosen. And the FBCMS2 results higher as compared to FBCMS1.

N.Taherkhani et al. (2016) developed a centralized and localized congestion management technique using the clustering approach to machine learning. Three units are working together to solve the problem. This technique consists of three components: congestion management, data control and channel congestion detection. The congestion management device uses a valid value parameter. Data from various resources and filters are stored in the control unit and grouped using a machine learning algorithm. Data congestion in the channel is observed in the congested detection unit. Then the measured values are eventually transmitted to the surrounding vehicle by RSU. This proposed scheme achieves improved performance over delay and packet error ratios. 
Khattab et al. (2017) QDA and Linear Discriminant Analysis defend VANAT from denial of service (DOS) and black-hole attacks. The actions of the malicious node are paying a great deal of attention. In the simulation, SUMO is created and supplied to NS2, a network simulator that provides the result through trace files. The technique suggested is used to collect pathological node data such as normal or abnormal. The method exposes the attack before it is produced.

The OLSR (optimized link state routing) was suggested by Abdel Waheb et al. (2013). It is one of MANET's renowned routing protocols. VANET QoS OLSR uses MPR (Multipoint Rely). MR is used for retransmission of the network packet, a form of the constructive protocol where each node has a path of each network node. In this overhead message protocol to keep the tracks, QOLSR is proposed high later, as it selects the optimum route for choosing the wireless Adhoc protocol. Abdel Wahab introduced other VANET QoS OLSR protocol in 2013. This protocol works for VANET as it can find ways in a rapid restriction of a shift in topology

A sophisticated clustering algorithm is proposed by Clausen T et al. (2001) and A. Temurnikar et al. (2020). The proposed work is based on PSO, which is used to create a stable cluster where four modules operate on the algorithm, NS2 and SUMO are used for simulation. The algorithm results show improved efficiency over the previous algorithm based on measured parameters such as production, packet delivery ratio, end-to-end delay, energy usage and overhead routing.

The new technique of clustering, based on a weighted algorithm (SVWCA) for the VANET, which is based on PDR (packet drop ratio), PMOR (packet modification rate), and PMIR (packet misroute rate), was adopted by A.Temurnikar et al. (2013). Any node importance in the network is calculated to identify a malicious node.

Trupil Limbasiya et al. (2018) proposed a secure authentication to measure total execution time for the discrete attack. Dhiman et al. (2020) and Pandey et al. (2020) applied an H-REVA algorithm based on the hypervolume concept. The result of the algorithm is better than the competitor algorithm. More Recent work proposed by Mukesh Soni et al. (2018) for Detection of Sybil attack of the network.

Khekare el al. (2013) proposed a smart city framework for intelligent traffic systems that avoid false messaging and provide actual traffic and weather conditions to drivers. Later on, Khekare et al. (2014) proposed an emergency messaging system to avoid an accident and alert drivers by emergency messaging. Kehekare et al. (2020) used an optimal path routing algorithm for VANET based on a reinforcement learning algorithm.

Mukesh Soni et al. (2018) has proposed a security scheme for remote user identification Mukesh Soni et al. (2021), given a lightweight message verification authentication scheme. Choudhary et al. (2021) has proposed a safety feature in an automatic vehicle that help in accident time, provide a location, and call to emergency number to save a life. Soni et al. (2021) also proposed a QUIC protocol for fast navigation on the internet, which builds on UDP. Soni et al. (2018) has given a brief study of the handle and simulate a large scale of Big data and also give the analysis of human movement identification using Wi-Fi signal.

Kaur et al. (2020) introduced an optimization algorithm named Tunicate Swarm Algorithm (TSA). This algorithm can solve real case study of search problem Garg et al. (2020), given a novel approach based on a neural network to detect defected surface, which can also be applied for Vehicles.

Dehghnani, Gaurav et al. (2020) presented an optimized algorithm based on a heuristic population named spring search algorithm (SSA), aiming to solve a critical problem related to single-objective constrained optimization, and also worked on a Multi-objective Seagull Optimization Algorithm (EMoSOA). This algorithm employs a dynamic archive concept, grid mechanism, leader selection, and genetic operators to cache non-dominated Pareto solutions. Later on, the idea of security based on face recognition is proposed, which can be used on VANET driver face recognition to check the driver's authority.

A novel HKN-RVEA and MoSEE algorithm was proposed by Gaurav et al. (2020), and the various work of optimization like rat swarm optimizer is proposed by author MOSHEPO a hybrid 


\begin{tabular}{|l|l|l|l|}
\hline Authors \& Years & Parameter Calculated & Simulation tools & Recent Modification in PSO \\
\hline $\begin{array}{l}\text { Manickavelu et } \\
\text { al. (2014) }\end{array}$ & $\begin{array}{l}\text { Delivery ratio, delay, } \\
\text { Packet drop Ratio }\end{array}$ & NS2 & $\begin{array}{l}\text { Algorithm for route recovery in } \\
\text { MANET }\end{array}$ \\
\hline $\begin{array}{l}\text { Kairwartya et al. } \\
(2015)\end{array}$ & Throughput and delay & MOVE & $\begin{array}{l}\text { Multi objective Dynamic Vehicle } \\
\text { Routing }\end{array}$ \\
\hline $\begin{array}{l}\text { Yassine Hernaf et } \\
\text { al. (2017) }\end{array}$ & $\begin{array}{l}\text { Simulation Time, Radio } \\
\text { Propagation }\end{array}$ & $\begin{array}{l}\text { MOVE, NS2, } \\
\text { SUMO }\end{array}$ & ACO and PCO Algorithm \\
\hline $\begin{array}{l}\text { Sadip Midya et } \\
\text { al. (2018) }\end{array}$ & $\begin{array}{l}\text { Service Delay, Mean } \\
\text { Square Error }\end{array}$ & $\begin{array}{l}\text { SUMO, NS3, } \\
\text { MATLAB }\end{array}$ & $\begin{array}{l}\text { A PSO optimized resource allocation in cloud } \\
\text { Architecture }\end{array}$ \\
\hline $\begin{array}{l}\text { Deepak } \\
\text { Rewadkar et al. } \\
(2019)\end{array}$ & $\begin{array}{l}\text { Traffic Density, End } \\
\text { to end delay, Fitness } \\
\text { function }\end{array}$ & SUMO, NS2 & $\begin{array}{l}\text { Traffic aware Routing in urban using PSO } \\
\text { model }\end{array}$ \\
\hline $\begin{array}{l}\text { Akthar Husain et } \\
\text { al (2020) }\end{array}$ & $\begin{array}{l}\text { Delivery ratio, delay, } \\
\text { Packet drop Ratio, } \\
\text { Throughput, Normalized } \\
\text { routing load }\end{array}$ & MATLAB & PSO Optimized Geocast Routing in VANET \\
\hline
\end{tabular}

multi-objective is applied to solve economic load dispatch problem. In the future, these techniques can be used in VANET to solve the critical issue.

Table 1 summarizes the related work base on PSO, which contributes to the VANET network. As shown in the table, Manickavelu et al. (2014) have proposed a method for MANET route discovery. Kairwartya et al. (2015) proposed a multi-objective dynamic vehicle algorithm that worked on the MOVE simulator. Later on, Yassine Hernaf et al. (2017) proposed an algorithm base on ACO and PSO simulated on MOVE, NS2, and SUMO. Sadip Midya et al. (2018) developed a PSO optimized resource allocation protocol based on cloud architecture. Traffic aware Routing in urban using PSO model suggested by Deepak Rewadkar et al. (2019) and PSO Optimized Geocast Routing in VANET proposed by Akthar Husain et al. (2020). Various parameters results have been calculated to give the best PSO based VANET algorithm.

\section{PROPOSED METHOD}

In existing work, every vehicle node sends the broadcast message to its nearby node vehicle to update details in the regular interval by hello message by this every node update their information table at regular intervals. Nodes can identify how much they are far from the hop. This mobility information of the node helps the Cluster member to choose the $\mathrm{CH}$ (Cluster Head). In this, unique work is proposed for the selection of cluster head based on PSO. A PSO enables a multi-hop approach that has been set up. Its job is explained below in the proposed algorithm. The entire work process is defined in the context of a proposed algorithm below (Fig 2).

We have tried to resolve the problem of clustering by devising this algorithm. In the proposed algorithm, the first step to form the network and region division. In the next step, the energy coverage area calculation of communication devices. Further probability calculation of network with dead and without dead sensor. The cluster setup with the help of the PSO mechanism. A vehicle with Max g_best Value selected as cluster head. Cluster head start the forwarding the data to RSU and nearby vehicle.

\subsection{Proposed Algorithm}

Step 1: Start

Step 2: Network formation 


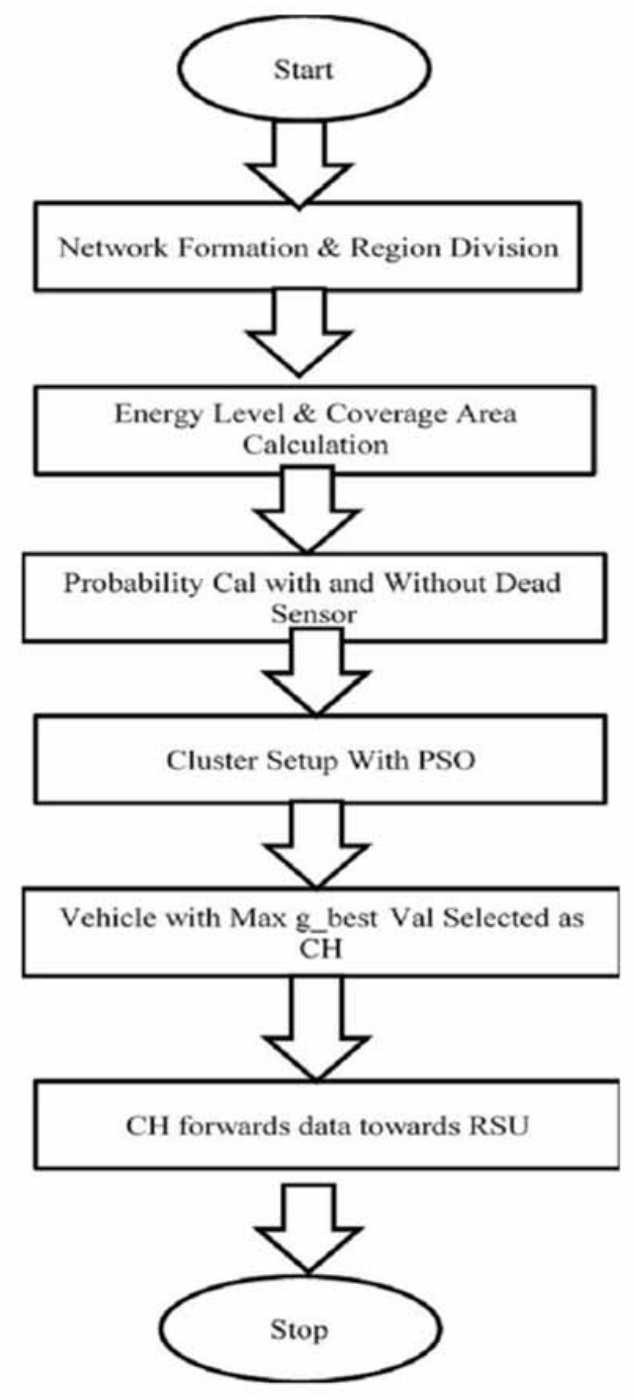

Step 3: Probability calculation with and without dead sensor

Step 4: Calculation of QoS and Distrust Value

Step 5: Making List of Honest and Dishonest Vehicle

Step 6: Cluster set up with PSO
A: Initialize vehicle
B: compute fitness of each vehicle

(i) If present fitness value is superior the pbest then

Pbest $=$ present fitness

(ii) gbest to greatest between all pbest

C: for each vehicle update position and velocity 
Step 7: Vehicle with max gbest selected as cluster head

Step 8: CH start communication with RSU and CM (Cluster Member)

Step 9: Stop

\section{RESULTS AND DISCUSSION}

For the simulation, the commonly used network simulator NS2 is used. Clustering V2V routing algorithm based on PSO ins proposed to improve the efficiency of the network. The algorithm comprises mainly three components first network formation, Probability calculation with and without dead sensor, PSO optimization, and $\mathrm{CH}$ (cluster head selection). The vehicle's different speed is considered in the simulation, and the simulation scenario is given in Table 1. After a simulation is performed, NS2 produces two files. Nam and.tr extensions. NAM files are used to visualize the simulation in NS2, as seen in figures, while the simulation outcome is evaluated using the.tr file. With the gnu plot, $x$ graph, or other methods, the result can be plotted. However, awk scripts were used to measure average throughput, average end-to-end delay, and packet transmission ratio for this project. Thus, data are plotted using LibreOffice Calc. The simulation screenshot is shown in Figure 3 , and Figure 4 is shown. Where PDR and throughput are calculated at a different speed. Various speed of a vehicle is considered for Proof of the proposed work. Figure 5 and Figure 6 shows the result calculated at a different rate in term of PDR and throughput. In Figure 5, PDR is calculated at a different rate where the $y$-axis shows the PDR, and the x-axis shows time. In Figure 6, throughput is calculated where the $y$-axis shows the throughput, and the $x$-axis shows the time.

\subsection{PDR (Packet Delivery Ratio)}

Packet Delivery ratio is ratio of data packets successfully delivered by total no of data packet generated. Mathematically representation of Packet delivery ratio.

$\mathrm{PDR}=\mathrm{PD} / \mathrm{PG}$

Where $\mathrm{PD}=$ Total number of packets delivered successfully, $\mathrm{PG}=$ Total number data generated

\subsection{Throughput}

The VANET performance is successfully obtained byackets in terms of kbps. Mathematically, the output can be represented as:

Throughput $=$ SP/TL

where SP is the total number of packets transmitted successfully, TL is the timestamp recorded in last transmitted packet.

To simulate realistic experimental conditions, we made the necessary adjustments, which are shown in the Table 2 . The simulation tools NS2 uses all mobile nodes with speed between $20 \mathrm{~m} / \mathrm{s}$ to $60 \mathrm{~m} / \mathrm{s}$ using a network size of $1200 \mathrm{~m} \times 600 \mathrm{~m}$.

Figure 3 is Initialization of Network on simulator NS2 where we have taken of 100 mobile nodes. A proposed multi-hop PSO algorithm works on it.

In Figure 4, the is communication between the mobile nodes. A Cluster head chosen with the help of PSO enables a multi-hop algorithm. It has been started to communicate with nearby nodes 
Table 2. Simulation Parameter

\begin{tabular}{|l|l|}
\hline Parameter & Value \\
\hline Simulation tools & NS2 \\
\hline Network Size & $1200 \mathrm{~m} \times 600 \mathrm{~m}$ \\
\hline Number Of Nodes & 100 \\
\hline Simulation time & $60 \mathrm{~s}$ \\
\hline MAC Protocol & IEEE 802.11 \\
\hline Vehicle Speed & $20,30,40,50,60 \mathrm{~m} / \mathrm{s}$ \\
\hline Protocol Used & AODV \\
\hline
\end{tabular}

Figure 3. Initialization of network

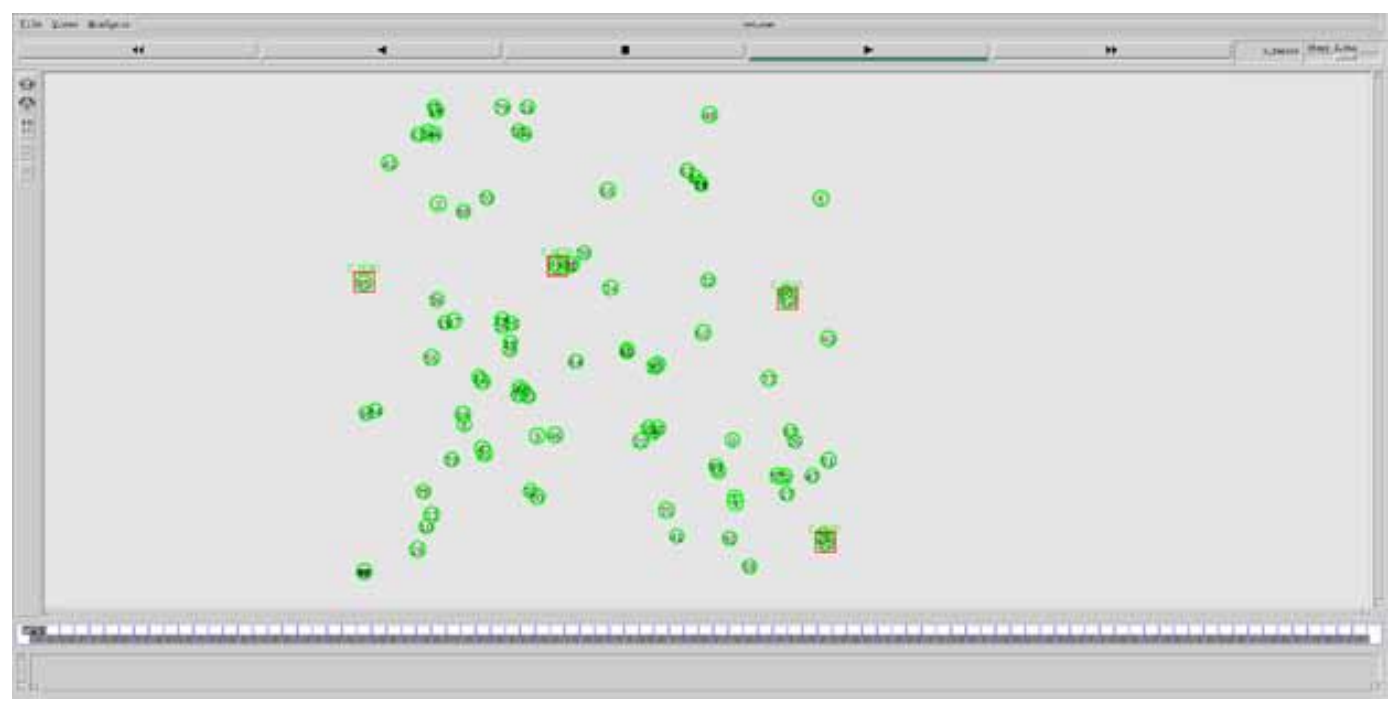

Figure 4. Data Communication of Network

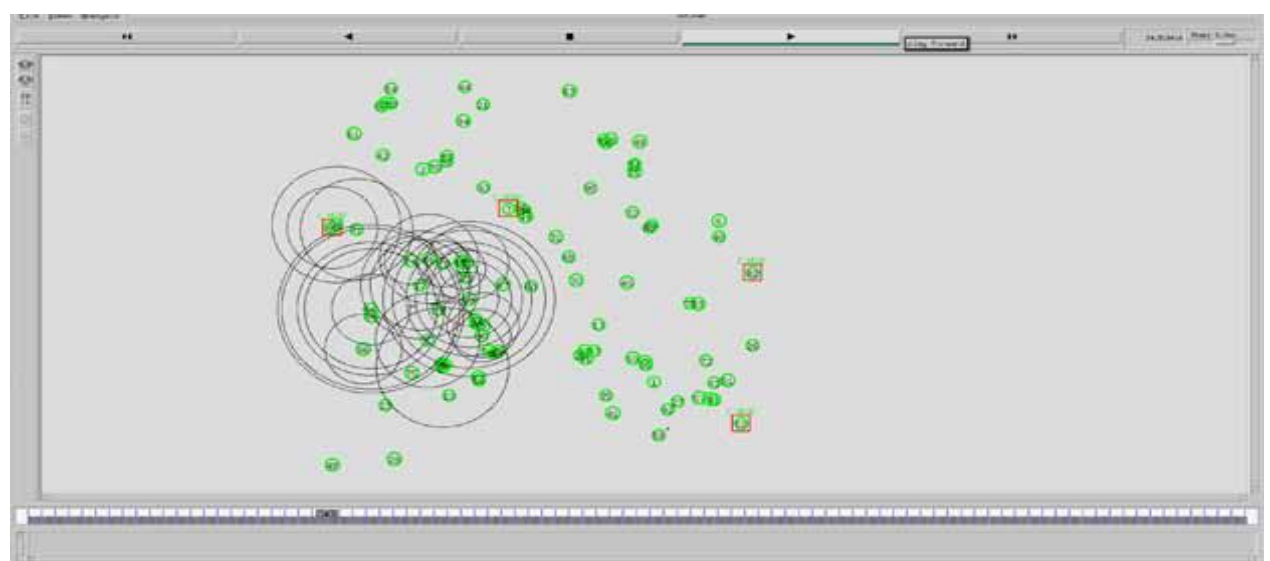




\section{Figure 5. PDR Vs Time}

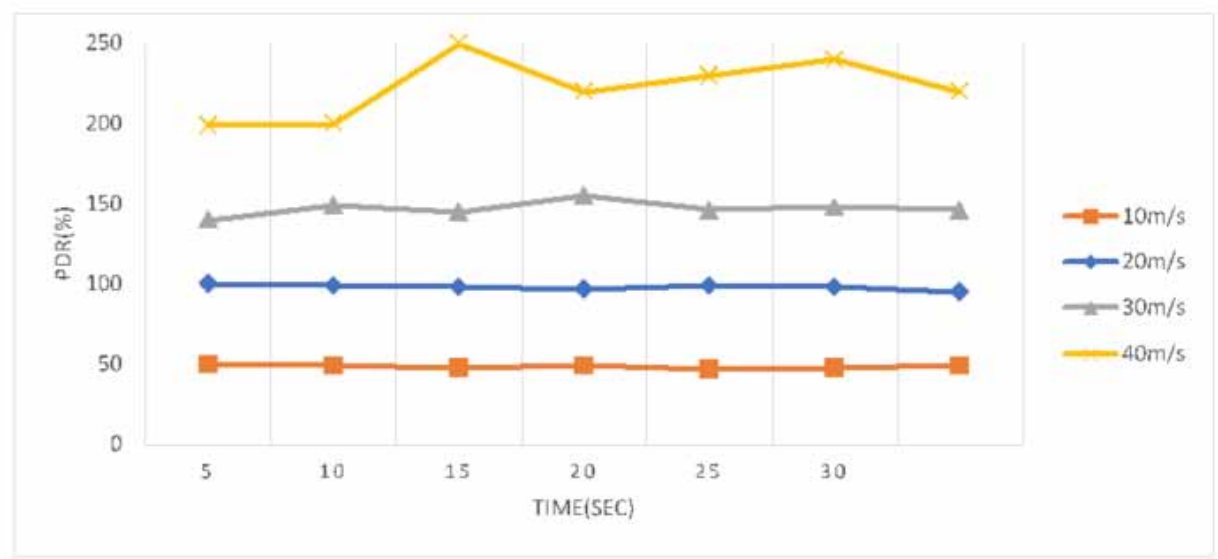

Figure 5. PDR Vs Time

Figure 6. Throughput Vs Time

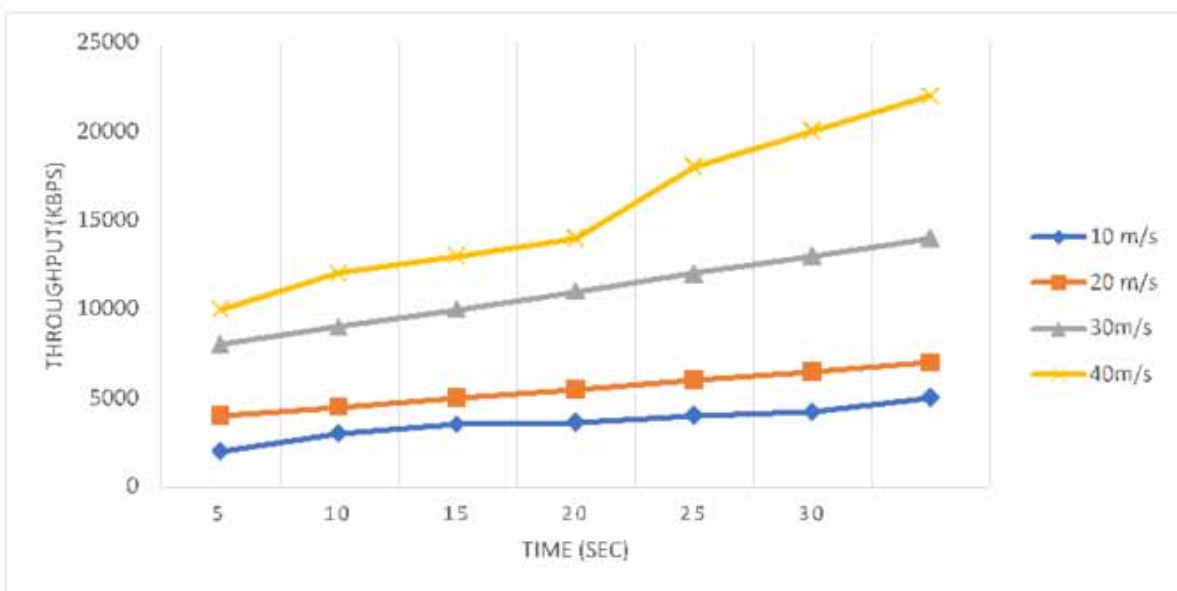

Figure 6. Throughput Vs Time 


\section{CONCLUSION}

In this paper, a novel PSO enable multi-hop approach has been proposed for clustering and security specially design for urban scenarios. NS2 simulator is used for simulation. SUMO allows to create traffic and generate random traffic. SUMO is a good simulation tool for VANET. In the simulation of proposed work, PDR and Throughput is calculated at a different speed to know robustness of the network. The result of proposed work is having $20 \%$ improvement over the previous outcome. The proposed method is used to overcome the existing problem and to enhance the performance of the network. 


\section{REFERENCES}

Alheeti, K. M., Gruebler, A., \& McDonald-Maier, K. (2017). undefined. Digital Communications and Networks, 3(3), 180-187. doi:10.1016/j.dcan.2017.03.001

Chaudhary, U., Patel, A., Patel, A., \& Soni, M. (2020). Survey paper on automatic vehicle accident detection and rescue system. Lecture Notes on Data Engineering and Communications Technologies, 319-324. 10.1007/978981-15-4474-3_35

Chaudhary, U., Patel, A., Patel, A., \& Soni, M. (2021). Survey Paper on Automatic Vehicle Accident Detection and Rescue System. In K. Kotecha, V. Piuri, H. Shah, \& R. Patel (Eds.), Data Science and Intelligent Applications. Lecture Notes on Data Engineering and Communications Technologies (Vol. 52). Springer. https://doi. org/10.1007/978-981-15-4474-3_35.

Comparison of ACO and PSO algorithm using energy consumption and load balancing in emerging Manet and VANET infrastructure. (2020). Journal of Critical Reviews, 7(9). 10.31838/jcr.07.09.219

Dehghani, M., Montazeri, Z., Dehghani, A., Samet, H., Sotelo, C., Sotelo, D., Ehsanifar, A., Malik, O. P., Guerrero, J. M., Dhiman, G., \& Ramirez-Mendoza, R. A. (2020). DM: Dehghani method for modifying optimization algorithms. Applied Sciences (Basel, Switzerland), 10(21), 7683. https://doi.org/10.3390/app10217683

Dehghani, M., Montazeri, Z., Dehghani, A., Samet, H., Sotelo, C., Sotelo, D., Ehsanifar, A., Malik, O. P., Guerrero, J. M., Dhiman, G., \& Ramirez-Mendoza, R. A. D. M. (2020). Dehghani Method for Modifying Optimization Algorithms. Applied Sciences (Basel, Switzerland), 10, 7683.

Dehghani, M., Montazeri, Z., \& Dhiman, G. (2020). A Spring Search Algorithm Applied to Engineering Optimization Problems. Applied Sciences (Basel, Switzerland), 10(18), 6173.

Dhiman, G. (2020). An Innovative Approach for Face Recognition Using Raspberry Pi. Artificial Intelligence Evolution.

Dhiman, G. (2020). MOSHEPO: A hybrid multi-objective approach to solve economic load dispatch and micro grid problems. Applied Intelligence, 50(1), 119-137.

Dhiman, G., \& Garg, M. (2020). MoSSE: A novel hybrid multi-objective meta-heuristic algorithm for engineering design problems. Soft Computing, 1-20.

Dhiman, G., Garg, M., Nagar, A. K., Kumar, V., \& Dehghani, M. (2020). A novel algorithm for global optimization: Rat swarm optimizer. Journal of Ambient Intelligence and Humanized Computing.

Dhiman, G., \& Kaur, A. (2019). HKn-RVEA: A novel many-objective evolutionary algorithm for car side impact bar crashworthiness problem. International Journal of Vehicle Design, 80(2-4), 257-284.

Dhiman, G., Oliva, D., Kaur, A., \& Singh, K. K. S. (2021). BEPO: A novel binary emperor penguin optimizer for automatic feature selection. Knowledge-Based Systems, 211. 10.1016/j.knosys.2020.106560

Dhiman, G., Oliva, D., Kaur, A., Singh, K. K., Vimal, S., Sharma, A., \& Cengiz, K. (2021). BEPO: A novel binary emperor penguin optimizer for automatic feature selection. Knowledge-Based Systems, 211, 106560. https://doi.org/10.1016/j.knosys.2020.106560

Dhiman, G., Singh, K. K., Slowik, A., Chang, V., Yildiz, A. R., Kaur, A., \& Garg, M. (2020). EMoSOA: A new evolutionary multi-objective seagull optimization algorithm for global optimization. International Journal of Machine Learning and Cybernetics, 1-26.

Dhiman, G., Soni, M., \& Pandey, H. M. (2020). A novel hybrid hypervolume indicator and reference vector adaptation strategies based evolutionary algorithm for many-objective optimization. Engineering with Computers. 10.1007/s00366-020-00986-0

Dhurandher, S. K., Singh, J., Woungang, I., Gupta, M., \& Sabharwal, N. (2019). Geometric shapes-based PSO approach for routing in vehicular networks. 2019 IEEE AFRICON. 10.1109/AFRICON46755.2019.9134043

El Khatib, A., Mourad, A., Otrok, H., Wahab, O. A., \& Bentahar, J. (2015). A cooperative detection model based on artificial neural network for VANET QoS-OLSR protocol. 2015 IEEE International Conference on Ubiquitous Wireless Broadband (ICUWB). doi:10.1109/ICUWB.2015.7324400 
Garg, M., \& Dhiman, G. (2020). Deep convolution neural network approach for defect inspection of textured surfaces. Journal of the Institute of Electronics and Computer, 2(1), 28-38.

Garg, M., \& Dhiman, G. (2020). Deep convolution neural network approach for defect inspection of textured surfaces. Journal of the Institute of Electronics and Computer, 2(1), 28-38.

Garg, M., \& Dhiman, G. (2020). A novel content-based image retrieval approach for classification using glcm features and texture fused lbp variants. Neural Compute Appl.

Hernafi, Y., Ben Ahmed, M., \& Bouhorma, M. (2017). ACO and PSO algorithms for developing a new communication model for VANET applications in smart cities. Wireless Personal Communications, 96(2), 2039-2075. https://doi.org/10.1007/s11277-017-4286-0

Husain, A., Singh, S. P., \& Sharma, S. C. (2020). PSO optimized Geocast routing in VANET. Wireless Personal Communications. 10.1007/s11277-020-07681-9

Husnain, G., Anwar, S., \& Shahzad, F. (2017). Performance evaluation of CLPSO and MOPSO routing algorithms for optimized clustering in vehicular ad hoc networks. $201714^{\text {th }}$ International Bhurban Conference on Applied Sciences and Technology (IBCAST). doi:10.1109/IBCAST.2017.7868141

Kadadha, M., Otrok, H., Barada, H., Al-Qutayri, M., \& Al-Hammadi, Y. (2018). A cluster-based QoS-OLSR protocol for urban vehicular ad hoc networks. In 2018 14th International Wireless Communications \& Mobile Computing Conference. IWCMC. doi:10.1109/IWCMC.2018.8450405

Kaiwartya, O., Kumar, S., Lobiyal, D., Tiwari, K. P., Abdullah, H. A., \& Hassan, N. A. (2015). Multiobjective dynamic vehicle routing problem and time seed-based solution using particle swarm optimization. Journal of Sensors.

Kaur, , \& Awasthi, , Sangal, \& Dhiman. (2020). Tunicate Swarm Algorithm: A new bio-inspired based metaheuristic paradigm for glob optimization. Engineering Applications of Artificial Intelligence, 103541.

Kaur, H., Rai, A., Bhatia, S. S., \& Dhiman, G. (2020). MOEPO: A novel Multi-objective Emperor Penguin Optimizer for global optimization: Special application in ranking of cloud service providers. Engineering Applications of Artificial Intelligence, 96. 10.1016/j.engappai.2020.104008

Kaur, Jain, Goel, \& Dhiman. (2020). A Review on Machine-Learning Based Code Smell Detection Techniques in Object-Oriented Software System(s). Recent Advances in Electrical \& Electronic Engineering, 13, 1. https:// doi.org/10.2174/2352096513999200922125839

Khekare \& Sakhare. (2013). A smart city framework for intelligent traffic system using VANET. 2013 International Mutli-Conference on Automation, Computing, Communication, Control and Compressed Sensing (iMac4s), 302-305. doi: 10.1109/iMac4s.2013.6526427

Khekare, Verma, Dhanre, Raut, \& Sheikh. (2020). The Optimal Path Finding Algorithm Based on Reinforcement Learning. International Journal of Software Science and Computational Intelligence, 12, 4. doi:10.4018/ IJSSCI.2020100101

Khekare, G. S. (2014). Design of emergency system for intelligent traffic system using VANET. International Conference on Information Communication and Embedded Systems (ICICES2014), 1-7. doi: doi:10.1109/ ICICES.2014.7033910

Kumar, G., Saha, R., Rai, M., \& Kim, T-h. (2018). Multidimensional Security Provision for Secure Communication in Vehicular Ad hoc Networks using Hierarchical Structure and End-to-End Authentication. IEEE Access. .10.1109/ACCESS.2018.2866759

Limbasiya, T., Soni, M., \& Mishra, S. K. (2018). Advanced formal authentication protocol using smart cards for network applicants. Computers \& Electrical Engineering, 66, 50-63. doi:10.1016/j.compeleceng.2017.12.045

Limbasiya, T., Soni, M., \& Mishra, S. K. (2018). Advanced formal authentication protocol using smart cards for network applicants. Computers \& Electrical Engineering, 50-63.

Manickavelu, D., \& Vaidyanathan, U. R. (2014). Particle swarm optimization (PSO)-based node and link lifetime prediction algorithm for route recovery in MANET. EURASIP Journal on Wireless Communications and Networking, 107. 
Midya, S., Roy, A., Majumder, K., \& Phadikar, S. (2018). PSO based optimized resource allocation in three tier cloud architecture for VANET. 2018 Fourth International Conference on Research in Computational Intelligence and Communication Networks (ICRCICN). 10.1109/icrcicn.2018.8718723

Nair, R., Gupta, S., Soni, M., Shukla, P., \& Dhiman, G. (2020). An Approach to Minimize the Energy Consumption during Blockchain Transaction. Materials Today: Proceedings.

Ozera, K., Bylykbashi, K., Liu, Y., \& Barolli, L. (2018). A fuzzy-based approach for cluster management in VANETs: Performance evaluation for two fuzzy-based systems. Internet of Things, 3-4, 120-133. doi:10.1016/j. iot.2018.09.011

Pandey, H. M., Dhiman, G., Soni, M., Slowik, A., \& Kaur, H. (2020). A Novel Hybrid Evolutionary Algorithm based on Hypervolume Indicator and Reference Vector Adaptation Strategies for Many-Objective Optimization. Engineering with Computers.

Rajawat, B. S., Tomar, R. S., Sharma, M. S., \& Chaurasia, B. K. (2018). Improved election of cluster head using CH-PSO for different scenarios in VANET. Communications in Computer and Information Science, 839, 110-120. doi:10.1007/978-981-13-2372-0_10

Rawat, D. B., Garuba, M., Chen, L., \& Yang, Q. (2017). On the security of information dissemination in the internet-of-Vehicles. Tsinghua Science and Technology, 22(4), 437-445. doi:10.23919/TST.2017.7986946

Rewadkar, D., \& Dove, D. (2019). Traffic-aware routing in urban VANET using PSO model. Journal of Networking and Communication Systems, 2(2). https://doi.org/10.46253/jnacs.v2i2.a4

Soni \& Kumar. (2020). Wavelet Based Digital Watermarking Scheme for Medical Images. 2020 12th International Conference on Computational Intelligence and Communication Networks (CICN), 403-407. doi: doi:10.1109/ CICN49253.2020.9242626

Soni, Chauhan, \& Puri. (2020). An Approach to Enhance Fall Detection Using Machine Learning Classifier. 2020 12th International Conference on Computational Intelligence and Communication Networks (CICN), 229-233. doi: doi:10.1109/CICN49253.2020.9242634

Soni \& Singh. (n.d.). Median First Tournament Sort. International Journal of Computer Science Engineering and Information Technology Research, 2(1), 35-52.

Soni, M., \& Jain, A. (2018). Secure Communication and Implementation Technique for Sybil Attack in Vehicular Ad-Hoc Networks. Second International Conference on Computing Methodologies and Communication (ICCMC), 539-543. doi: doi:10.1109/ICCMC.2018.8487887

Soni, M., Jain, A., \& Patel, T. (2018). Human Movement Identification Using Wi-Fi Signals. 2018 3rd International Conference on Inventive Computation Technologies (ICICT), 422-427. doi: doi:10.1109/ ICICT43934.2018.9034451

Soni, M., \& Patel, T. (2018). Systematic investigation on Large Scale simulations in big data systems. 2018 2nd International Conference on Inventive Systems and Control (ICISC), 684-688. doi: doi:10.1109/ ICISC. 2018.839888

Soni, M., Patel, T., \& Jain, A. (2020). Security Analysis on Remote User Authentication Methods. In A. Pandian, T. Senjyu, S. Islam, \& H. Wang (Eds.), Proceeding of the International Conference on Computer Networks, Big Data and IoT (ICCBI - 2018). ICCBI 2018. Lecture Notes on Data Engineering and Communications Technologies (Vol. 31). Springer. https://doi.org/10.1007/978-3-030-24643-3_60.

Soni, M., \& Rajput, B. S. (2021). Security and Performance Evaluations of QUIC Protocol. In K. Kotecha, V. Piuri, H. Shah, \& R. Patel (Eds.), Data Science and Intelligent Applications. Lecture Notes on Data Engineering and Communications Technologies (Vol. 52). Springer. https://doi.org/10.1007/978-981-15-4474-3_51.

Soni, M., Rajput, B. S., Patel, T., \& Parmar, N. (2021). Lightweight Vehicle-to-Infrastructure Message Verification Method for VANET. In K. Kotecha, V. Piuri, H. Shah, \& R. Patel (Eds.), Data Science and Intelligent Applications. Lecture Notes on Data Engineering and Communications Technologies (Vol. 52). Springer. https:// doi.org/10.1007/978-981-15-4474-3_50.

Taherkhani, N., \& Pierre, S. (2016). Centralized and localized data congestion control strategy for vehicular ad hoc networks using a machine learning clustering algorithm. IEEE Transactions on Intelligent Transportation Systems, 17(11), 3275-3285. doi:10.1109/TITS.2016.2546555 
Temurnikar, A., Verma, P., \& Choudhary, J. (2020). Securing vehicular Adhoc network against malicious vehicles using advanced clustering technique. 2nd International Conference on Data, Engineering and Applications (IDEA). doi:10.1109/IDEA49133.2020.9170696

Wahab, O. A., Mourad, A., Otrok, H., \& Bentahar, J. (2016). CEAP: SVM-based intelligent detection model for clustered vehicular ad hoc networks. Expert Systems with Applications, 50, 40-54. doi:10.1016/j.eswa.2015.12.006

Wahab, O. A., Otrok, H., \& Mourad, A. (2013). VANET QoS-OLSR: Qos-based clustering protocol for vehicular ad hoc networks. Computer Communications, 36(13), 1422-1435. doi:10.1016/j.comcom.2013.07.003

Yuvaraj, N., Srihari, K., Chandragandhi, S., \& Arshath Raja, R. (2020). Gaurav Dhiman, and Amandeep Kaur. "Analysis of protein-ligand interactions of SARS-Cov-2 against selective drug using deep neural networks. Big Data Mining and Analytics.

Ankit Temurnikar is a PhD student of Computer Science Engineering at Bhagwant University Ajmer Rajasthan. He has been a researcher in VANET since 2012. His current research is concerned with VANET clustering and security. He has worked in School of Research and Technology Peoples University Bhopal from 2013 to 2018.

Pupspneel Verma $(P h D)$ received PhD Degree from Bhagwant University. He is working as associate professor in Bhagwant University his area of research are Computer Network, MANET, VANET, etc. 